Canadian University Music Review

Canadian University Music Review

Revue de musique des universités canadiennes

\title{
Quel plain-chant pour la Nouvelle-France? L'exemple des Ursulines
}

\section{Jean-Pierre Pinson}

Volume 11, numéro 2, 1991

URI : https://id.erudit.org/iderudit/1014105ar

DOI : https://doi.org/10.7202/1014105ar

Aller au sommaire du numéro

Éditeur(s)

Canadian University Music Society / Société de musique des universités canadiennes

ISSN

0710-0353 (imprimé)

2291-2436 (numérique)

Découvrir la revue

Citer cet article

Pinson, J.-P. (1991). Quel plain-chant pour la Nouvelle-France? L'exemple des Ursulines. Canadian University Music Review / Revue de musique des universités canadiennes, 11(2), 7-32. https://doi.org/10.7202/1014105ar

(c) Canadian University Music Society / Société de musique des universités canadiennes, 1991
Ce document est protégé par la loi sur le droit d'auteur. L'utilisation des services d'Érudit (y compris la reproduction) est assujettie à sa politique d'utilisation que vous pouvez consulter en ligne.

https://apropos.erudit.org/fr/usagers/politique-dutilisation/ 


\title{
QUEL PLAIN-CHANT POUR LA NOUVELLE- FRANCE ? L'EXEMPLE DES URSULINES
}

\author{
Jean-Pierre Pinson
}

\section{Introduction}

S'il est une musique qui fut pratiquèe en Nouvelle-France depuis les origines jusqu'au $\mathrm{XX}^{\mathrm{e}}$ siècle, ce fut bien celle du plain-chant ${ }^{1}$. Or, ce répertoire se présente comme un reflet souvent exact du plain-chant gallican, de son histoire et de sa pratique. Certes, il lui a fallu s'adapter aux lieux, aux populations et aux coutumes, mais sa répartition géographique, moins complexe qu'en France, et le nombre plus restreint d'institutions et de paroisses permettent une methode d'approche à peu près contrôlable. Au cours de notre projet de recherche ${ }^{2}$. nous avons recueilli à travers le Quèbec des centaines de sources qui tracent un portrait riche de cette pratique. Mieux : au-delà du plain-chant lui-même c'est en fait le chant baroque français qui s'est conservé longtemps ici, « sous cloche », dans un milieu très conservateur : ce chant de la Nouvelle-France est un beau cas de Performing practice. Parmi toutes les institutions visitées, les Ursulines de Québec et de Trois-Rivières ${ }^{3}$ constituent une excellente illustration de ce phénomène. Les sources conservées chez elles, classées dans l'annexe bibliographique, présentent une synthèse assez fidèle des sources demeurées en Nouvelle-France, contribuant ainsi à l'elaboration de ce portrait.

\section{HISTORIQUE : «IL S'EN EST FALLU DE PEU QUE NOTRE CHANT N'AIT ÉTÉ RETRANCHÉ »}

L'histoire mouvementée du plain-chant chez Ursulines illustre bien le premier principe : la Nouvelle-France est un milieu conservateur.

1 Par « plain-chant », je désignerai le chant ecclésiastique qui suit le Concile de Trente, par opposition au « chant grégorien » du vieux fonds médiéval.

2 «Le plain-chant en Nouvelle-France, des origines au milieu du XIX ${ }^{e}$ siècle », projet subventionné par le Conseil de recherche en sciences humaines du Canada et la Faculté des arts del'Université Laval. Je tiens à remercier vivement les Ursulines de Trois-Rivières et de Québec, de même que Sœur Germaine Blais et Mme Christine Turgeon, archivistes, et Micheline Vézina-Derers, Musicologue, pour leur indispensable collaboration.

${ }^{3}$ Le couvent de Québec fut fondé en 1639; celui de Trois-Rivières, fondé en 1697, en sera dépendant jusqu'en 1731. 


\section{Les constitutions : 1639-1682}

Les Ursulines de France, au XVII ${ }^{e}$ siècle, sont réparties entre plusieurs communautés autonomes, aux constitutions particulières : Bordeaux, très liée à Tours, Paris, Bourges, Clermont-Ferrand, etc. Les Constitutions et coutumier de Bordeaux et de Tours de 1635, repris fidèlement par les Statuts de Tours de 1661, font une bonne place au chant liturgique que Marie de l'Incarnation, venue de Tours, dut amener avec elle en Nouvelle-France. Les chapitres sur la musique seront repris en grande partie dans les Constitutions que le Père Jérôme Lalemant, supérieur des Jésuites de Nouvelle-France, rédige (certainement en collaboration avec Marie de l'Incarnation) en 1647. Cependant, l'approbation de ces constitutions par Mgr de Laval, premier évêque de Québec, apporte une sérieuse restriction en supprimant :

le chant pendant la consécration à la sainte messe qui ne sera plus en usage; quelques parties des grandes messes qui se chantoient en plein-chant qui se diront doresnavant toutes entières à voix droite [...] les expositions du Saint-Sacrement au premier dimanche du mois et autres fêtes y spécifiées [sic] qui cesseront hors celles qui se trouveront dans l'octave du SaintSacrement.

Jusqu'à cette date, les Ursulines de Québec ont donc dû chanter comment elles le faisaient à Tours ou à Bordeaux. C'est au cours de cette période qu'apparaissent les premières allusions aux motets et à la musique instrumentale au monastère de Québec ${ }^{4}$.

La fin de cette période est signalée par la lettre bien connue de Marie de l'Incarnation du 13 septembre 1661, où elle fait allusion aux restrictions de Mgr de Laval :

Il s'en est fallu de peu que notre chant n'ait été retranché. Il nous laisse seulement nos vêpres et nos Ténèbres, que nous chantons comme vous faisiez au temps où j'étais à Tours. Pour la grand'messe, il veut qu'elle soit chantée à voix droite, n'ayant nul égard à ce qui se fait soit à Paris soit à Tours, mais seulement à ce que son esprit lui suggère être pour le mieux. Il craint que nous ne prenions de la vanité en chantant et que nous ne donnions de la complaisance au dehors. Nous ne chantons plus aux messes, parce que, dit-il, cela donne de la distraction au célébrant, et qu'il n'a point vu cela ailleurs (Oury $1971: 653)^{5}$.

4 Voir en particulier : Erich Schwandt, 1981 et 1984/1 et 2.

5 Les Annales, rédigées de mémoire à partir de 1700 (l'incendie de 1686 ayant détruit une bonne partie des livres et manuscrits) rappellent ce fait :

L'an 1642 le 21 de novembre nos premières mères prirent possession de notre premier monastère 
En 1682, Mgr de Laval annule les constitutions de 1647 pour les remplacer par celles des Ursulines de Paris. Le chant se limitera au chant des psaumes et des hymnes. Le chœur n'aura plus qu'à " dire le grand Office » durant les fêtes (comme celles de la Vierge) dont la liste est fournie. «Le chant sera d'un ton tout droit et sans notes $"{ }^{6}$.

\section{Après 1682 : le retour à la musique}

Cependant les choses changent en 1700. Les Annales, en témoignent :

Ce mesme iour [fin mars 1700] $\mathrm{Mon}^{\mathrm{r}}$ Glandelet ayant faict appeller les discretes ${ }^{7}$ a la sacristie leur desclara que dans la visite de Sup ${ }^{r}$ quil avoit faite au commencement de lannée quelques vunes luis avoient demandé quil plut a monseig ${ }^{r}{ }^{s}{ }^{s}$ donner le chant gregorien ce qui Surprit fort car la plus part de celles qui estoient presente ny penssoient nullement ${ }^{8}$.

Charles Glandelet est alors supérieur des Ursulines, vicaire général, chanoine et chantre au Chapitre de la cathédrale de Québec. Mgr de Saint-Vallier a succédé à Mgr de Laval. Les Annales précisent :

Il dit que monSeigr ny luy nestoient pas de Sentiment que no dussions entreprendre de chanter tout mais Seulement quaux $g^{\text {des }}$ messe lon shantats le Kirie le Gloria, le Credo, et le Sanctus et lagnus dei; et pour les vespres il ne Iuget pas quon dut chanter les pseaumes et les himnes en plain chant que les antiennes et les reste se diroient en chant droit a lordinaire.

On retourne donc au chant de l'ordinaire des « grandes fêtes » et à un chant des Vêpres plus modeste, la psalmodie recto tono (« chant droit ») renvoyant à la « voix droite » de Marie de l'Incarnation) ${ }^{9}$. Voilà aussi l'apparition des cours de plain-chant :

\footnotetext{
[...] avec un chœur pour les religieuses et l'on y chanta en ce jour la première messe [...] dévotion que l'on a continué plus de 18 ans durant et que l'on aurait continuée toujours si Mgr de Laval Evêque de Québec ne nous eut ordonné de discontinuer de chanter cette grande messe aussi bien que celle de saint Ignace dont nous faisions la fête au mois de juillet...

${ }^{6}$ L'approbation de l'évêque de Clermont de 1681, dans l'exemplaire conservé à Québec, précise même : "Quant au chant en notes, on n'en usera jamais sinon pour ce qui est marqué dans la liste". On remarquera que cette "liste de ce qu'on peut chanter en notes de plain-chant" est beaucoup plus restreinte qu'à Tours.

7 Religieuses conseillères de la Supérieure.

8 Annales, p. 99.

9 E. Schwandt relève dans les Annales qu'en octobre 1690 les Ursulines chantèrent le salut, les premières et secondes Vêpres de la Sainte-Ursule (Schwandt 1984/2 : 9)
} 
Ensuite lon commenca destrudier la note [apprendre le solfège] et mons ${ }^{r}$ Glandelet procura quun des messieurs du Seminaire vint 2 ou trois [fois] la $\mathrm{Sep}^{\text {ne }}$ [semaine] nous montrer une heure durant [;]nous fusmes en moins de dix Iours en estat de commencer la Samedy de chanter ce qui nous estoit prescrit por les messes reseruant a chanter les vespres en plain chant le iour de la feste du Sacre coeur que nous devons commencer cette année a la Celebrer avec $\mathrm{g}^{\mathrm{de}}$ solemnité le vendredi dapres loctave du tres $\mathrm{s}^{t}$ Sacrement pour continuer a lauenir chasque année a mes iour; cette solemnité consiste a lexposition du tres $S^{t} S a c r e m e n t$ Messe Solemnelle avec Diacre et soudiacre vespres et sermon avec le Salut mais lon de Sest pas voulu charger du $\mathbf{g}^{\mathrm{d}}$ office cest a dire des $\mathbf{g}^{\text {des }}$ matine MonSeig ${ }^{r}$ de Kebec en a donné la patente et en a approuué loffice qui en a esté composé par mon ${ }^{\mathrm{r}}$ Glandelet auec la messe propre permettant a tous les prestes seculiers et reguliers de son Dioceze den dire loffice et la messe. ${ }^{10}$

Les Annales précisent enfin cette nouvelle attitude :

Lon a commencé a chanter les Pseaumes de vespres en plain chant la veille de lascension laissant les tons figurés que no ${ }^{s}$ avions tousiours chantée depuis 60 ans que la maison a esté establie lon acommence ce mesme [jour?] a chanter en plain chant les complies.

Mgr de Saint-Vallier officialise (impose) donc le retour au plain-chant. Les religieuses reçoivent un enseignement musical. La mention de la fête du SacréCœur, dont la célébration commence en 1700 , donne un sens aux manuscrits conservés aux archives, qu'elle permet ainsi de dater. Les sœurs ne se sentent pas prêtes pour les " grandes matines ", qui sont complexes, mais ce n'est que partie remise. Glandelet fournit le matériel : Les archives des Ursulines de Québec possèdent un Catalogue des livres conservés dans les armoires qui signale des "Leçons de chant contenant la messe et les vêpres du Sacré-Cœur de Jésus par Messire Glandelet, V.[icaire] G.[énéral de Québec, 1700 " ". Enfin, cet office est prévu pour l'ensemble des paroisses du diocèse (qui couvre toute la NouvelleFrance d'alors), ce qui l'officialise définitivement.

Le retour au plain-chant se vérifie lors de fêtes propres à l'ordre. D'après les Annales, en 1752 Mgr de Pontbriand approuve les modifications de Mgr de Saint-Vallier. Le 19 mars 1768, fête de Saint Joseph. « la Messe a été Chantée en Plain Chant avec Permission de sa Grandeur ». En 1769, à propos de la

\footnotetext{
10 Annales, p. 99-100.

11 L'expression «composée » prend une importance particulière : on la retrouve à propos de la messe et de l'office de la Sainte-Famille, qui aurait été « composée », c'est-a-dire, en fait, « arrangée " par Charles-Amador Martin, chanoine, musicien et chantre du même Chapitre, et « collègue " de Glandelet.
} 
béatification d'Angèle de Mérici (fondatrice de l'ordre des Ursulines) le même evêque " a permis à la Communauté de chanter le plain-chant " (grand'messe et Te Deum). En 1788, les religieuses ont retrouvé le chant et même la polyphonie. Lors des obsèques de Mgr d'Esglis « Nous fîmes un Service Solennel et une Communion [...], nous chantâmes la prose et le libera en quatre parties et le Peccantem en Musique en deux parties a l'élévation... ». Douze ans plus tard paraîtront les premiers livres de chant imprimés à Québec, diffusés dans tout le diocèse, et bien représentés chez les Ursulines.

Il faut enfin souligner le rôle que la fête de la Sainte-Famille, propre à la Nouvelle-France, va jouer chez les Ursulines au XVIII ${ }^{e}$ siècle. La congrégation est fondée en 1664, mais, si on en croit Adrien Pouliot ${ }^{12}$, la messe et l'office de cette fête, approuvé en 1684 par Mgr de Laval, trouveront leur version définitive dans les années 1696-1702. Les Ursulines conservent une douzaine de manuscrits de la musique ${ }^{13}$.

Après une périods d'austérité (1661-1700) où la psalmodie a prévalu, la musique des Ursulines, en tout cas le plain-chant, a retrouvé au cours du XVIII' siècle une bonne partie de ses droits, même si les Annales parlent avant tout d'un nombre restreint de fêtes.

\section{LES SOURCES MUSICALES}

\section{Le répertoire dans les constitutions : cérémonies et fêtes}

Dans cette congrégation vouée en particulier à l'enseignement, le chapitre « De la distribution des heures du jour pour l'occupation des pensionnaires et séminaristes " prévoit le chant et la récitation. Les religieuses devront par exemple faire «dire » par les élèves les litanies et les Vêpres de la Vierge, faire « chanter » « quelquefois » des saluts «pour diverses nécessités » ou « tous les samedis pour les bienfaiteurs et bienfaitrices du séminaire ».

Les chapitres « De la distribution du temps et des occupations ou emploi de la journée » et « De l'oraison vocale » des constitutions de 1647 font le relevé des principales cerémonies : messes (" dimanches et fêtes ordinaires", fêtes de seconde et première classe, avec bref motet à l'élévation), litanies, chapelet, heures dont le petit office de la Vierge (récité), grand office des fêtes et des dimanches, dont les Vêpres et les complies, Ténèbres, exposition du SaintSacrement (un salut avec chant le premier dimanche de chaque mois). D'autres

12 Adrien Pouliot : 1980.

13 Charles Glandelet a joué un rôle important durant cette période d'élaboration, aux côtés de Charles Amador Martin (Pouliot 1980). 
chapitres signalent la rénovation des vœux et l'office des morts. La cérémonie des vêtures et des professions complète les cérémonies chantées.

Les constitutions de Bordeaux-Tours de 1635, et Tours 1661, donnent la liste des fêtes chantées : Nativité, Circoncision, Épiphanie, Pâques, Ascension, Pentecôte, Saint-Sacrement, Trinité, Toussaint, fêtes de la Vierge, auxquelles il faut joindre les fêtes de saint Jean-Baptiste, de saint Pierre et saint Paul « et les trois jours des Ténèbres ». Les Annales ajoutent les fêtes chantées du 21 novembre et de la Saint-Ignace du mois de juillet. Viennent enfin les fêtes propres a l'ordre : à Trois-Rivières, ces fêtes, depuis 1697, comprennent les offices de Noël (précédés de la neuvaine dite des « $\mathrm{O}$ »), du Sacré-Cœur, de saint Augustin, de sainte Ursule, de saint Joseph et de sainte Angèle ${ }^{14}$. Les constitutions de Paris (1673), qui s'en tiennent à la récitation, ajoutent quelques autres fêtes comme la Dédicace et le patron de l'église.

\section{Première période : 1642 (1639 ?)-1700 : quelle musique?}

Il faut sans doute imputer à l'incendie du monastère de Québec, en 1686, la disparition de la quasi totalité des sources musicales de la première période. On devra donc s'en tenir à des hypothèses fondées tant sur la pratique musicale dans les couvents de femmes français que sur les sources plus tardives conservées à Québec.

Les archives des Ursulines de Québec conservent deux graduels : un Graduale romanum, Paris : Chr. Ballard 1697 (annexe bibliographique $n^{\circ} 21$ ), qui appartint à une Ursuline de Québec. Ce recueil est appouvé par H. Dumont et Robert (1682), avec censure de Nivers (1697). Un autre Graduale romanum (Paris, 1706) $\left(\mathrm{n}^{\circ} 22\right)$ possède une censure de Nivers. La préface des Cantus diversi de Trois-Rivières (Lyon, 1643$)^{15}$ (no 23 ) nous avertit que cette réédition a été

augmentée de l'office des Morts, de deux Messes de M. Dumons [sic] [les très populaires Messe Royale du premier ton et messe du 2e ton], et des leçons de Jeremie de Mr de Nevers [Nivers]. (Voir exemple 1)

Par ailleurs, les deux plus anciens antiphonaires retrouvés à ce jour datent de 1666 et 1671 ( $\mathrm{n}^{\text {os }} 25$ et 26). Le premier est de la même veine que le graduel de 1697 (format, notation, typographie). Le second, qui porte la mention manuscrite «Aux Ursulines de Québec », n'est autre que l'Antiphonarium romanum de

14 Mère Sainte-Thérèse de Lisieux : 1961.

15 Combiné du graduel et de l'antiphonaire, formule très populaire au Québec jusq'au XX ${ }^{\mathrm{e}}$ siècle. 
Nivers, auquel ont été ajoutées à la main des pièces comme l'introït Gaudeamus (fêtes de la Vierge et de la Sainte-Famille) et la séquence de la Sainte-Famille. À ces recueils, il faut joindre les Vêpres de l'Avent $\left(\mathrm{n}^{0} 1\right)$, manuscrit de toute évidence copié sur un original imprimé et qui, malgré la présence des Vêpres de la Sainte-Famille (plus tardifs), semble remonter à une date antérieure. (Exemple 2)

On reconnaîtra ici deux types de livres français, dont certains pouvaient être utilisés par les communautés féminines françaises : un « corpus Nivers I » et un « corpus Nivers II ». Le premier corpus est représenté par les ouvrages de petit ou moyen format, et approuvé par Nivers, Dumont, Robert. Les mélodies sont traditionnelles, quoique simplifiées, le Concile de Trente aidant. Le corpus « Nivers II » regroupe les compositions originales en plain-chant musical mesuré et orné, dues à Nivers lui-même ${ }^{16}$.

Ces ouvrages sont plus tardifs que la période 1642-1662, mais ils relèvent d'une tradition caractéristique :

- L'Avertissement des Cantus diversi de $1643\left(\mathrm{n}^{\circ} 23\right)$ destine ce recueil aux « curés de campagne » et aux chœurs des paroisses « où tous les Ecclesiastiques ne peuvent pas se tenir devant un seul lutrin », ce qui est le cas d'un chœur de religieuses.

- Le répertoire « utilitaire » comprend en particulier le Kyrial et les fêtes signalées par les constitutions des Ursulines de Québec, y compris l'Office des Morts et, bien sûr, les Ténèbres.

- La notation est mesurée (longues à queue, carrées, losanges), avec le seul si bémol et des barres séparant les mots.

- L'ornementation est intéressante : l'antiphonaire de Nivers présente de nombreux agréments (croix et liaisons). La mélodie des Lamentations est remplie de losangées qui font œuvre de port-de-voix. Si ces agréments sont absents des graduels de Québec, on peut facilement les restituer aux places habituelles (après les ports-de-voix, aux " cadences »). Les messes de Dumont (plain-chant musical) se prêtent bien à cette pratique (d'autres versions retrouvées en Nouvelle-France en donnent l'ornementation).

Il semble donc qu'au milieu du XVII ${ }^{e}$ siècle, et à l'image des couvents féminins français, les Ursulines de Québec connaissaient tous les degrés du plain-chant, depuis la psalmodie jusqu'au plain-chant musical orné.

16 Pour la liste des œuvres de Nivers, voir Pruitt 1973, qui (p. 140) ne signale pas cette édition de 1671. 


\section{Le répertoire au $X V I I I^{e}$ siècle}

Les sources retrouvées aujourd'hui peuvent être rangées selon les catégories exposées ci-dessous $(\mathrm{a} \text { à } \mathrm{f})^{17}$.

\section{a. Manuscrits : offices divers}

Ces manuscrits sont de petit format et de mains diverses, et destinés aux fêtes propres aux Ursulines. Leur contenu va du plain-chant proprement dit au plainchant musical. Dans l'annexe bibliographique, nous proposons une datation fondée en particulier sur le nom des religieuses apparaissant sur les premières pages.

Ces manuscrits ont été utilisés (noms de religieuses, ajouts, corrections, pages usées, pages manquantes). Même si leur contenu n'est pas parfaitement homogène, certaines séries sont cohérentes. Ainsi, les différentes versions de l'office et de la messe de la Sainte-Famille sont très voisines, et très semblables àl'ensemble du corpus relevé à ce jour en Nouvelle-France. On noteral'apparition d'un répertoire en plain-chant musical orné, important pour l'étude du chant chez les Ursulines : ainsi la Messe Bordeloise (ordinaire), très ornée, est parfois jointe au propre de la messe du Sacré-Cœur. Un manuscrit $\left(\mathrm{n}^{\circ} 8\right)$ comprend la messe du 6e ton et la Messe Bordeloise, et présente même une page en notation " profane » sur 5 lignes qui n'est autre que la Musette en rondeau des Pièces de clavecin (1724) de Rameau, transposée en sol majeur, avec des paroles en français.

\section{b. Messe et office de la Sainte-Famille}

Très voisine des fêtes de saint Joseph et du Sacré-Cœur, cette fête fait appel aux différents types de plain-chant : l'office comprend les psaumes avec leurs antiennes, une antienne du Magnificat, des hymnes. L'ordinaire de la messe est la Messe Royale de Dumont, en plain-chant musical. (Exemple 3)

\section{c. Vêtures et professions}

Les couvents de Québec et de Trois-Rivières ne semblent conserver que le cérémonial des Ursulines de Paris. Dans toutes les versions retrouvées le texte est identique. Sans doute copiés sur un original venant de Québec après l'incendie du couvent de Trois-Rivières en 1806, deux manuscrits de mains

17 Il faut distinguer les sources contemporaines des sources qui ont pu être amenées plus tard (envois des Ursulines françaises en relation avec la Nouvelle-France, sources amenées par les religieuses du moins, tant que les nouvelles religieuses vinrent de France -, achats dont ceux de Thomas Maguire effectués auprès des Ursulines de Paris en 1835. 
différentes mais de contenus identiques donnent la version complète des chants des vêtures et des professions (Cérémonial des vêtures et professions, Paris 1716 [en fait : 1625] $\left(\mathrm{n}^{\circ} 16\right)$. La musique en est simple (les hymnes Veni Creator ou Te Deum, l'antienne Veni sancte Spiritus, par exemple, sont simplifiées); elle est souvent basée sur des timbres, à la manière des cantiques. La notation est faite de carrées et de losangées, impliquant des rythmes pointés ou ternaires, les accidents comprennent le sib et le do\#, de brèves liaisons indiquent parfois des ports-de-voix. (Exemple 4)

\section{d. Sépultures}

Cet office est doté d'une importance particulière (voir nos 19 et 20).

\section{e. Imprimés}

Nous avons déjà signalé certaines sources utiles à la compréhension du chant au cours de la première période. En se reportant à l'annexe bibliographique, le lecteur remarquera l'importance de la tradition des livres approuvés ou composés par Nivers, la présence des livres de Vannes et de Lyon, qui vont servir de références aux premières éditions québécoises du début du $\mathrm{XIX}^{\mathrm{e}}$ siècle.

\section{f. Méthodes}

Les méthodes conservées chez les Ursulines sont parmi les plus intéressantes du $\mathrm{XVIII}^{\mathrm{e}}$ siècle. Aucune preuve d'utilisation n'a cependant été relevée. Leur contenu suit un plan semblable. La Nouvelle méthode pour apprendre le plainchant (Paris : Ballard, 1683) $\left(\mathrm{n}^{\circ} 30\right)$ discute la question des valeurs (longues avec hampe, carrées, losangées) jointe à celle de la « quantité » de la prosodie latine. Cette question, rendue confuse par les multiples erreurs ou variantes des livres de chant, est une des plus complexes depuis les premières éditions qui ont suivi le Concile de Trente. La cohérence entre les multiples sources (méthodes et recueils de chant) est loin d'être parfaite, d'autant plus que la pratique ne semble pas vouloir toujours suivre la théorie. Cette Nouvelle méthode, consciente du problème prend le parti de la quantité :

Mais on doit plutost avoirégard en chantant aux regles de la Quantité qu'aux notes longues et brèves, parce que tous les livres sont pleins de fautes, et qu'il y a la plupart du temps des notes brèves sur les syllabes longues et des notes longues sur des syllabes brèves (p. 29).

Les Trois méthodes de 1713 qui accompagnent les Cantus diversi (Paris, 1643, AUTR) ( $\left.n^{\circ} 23\right)$ présentent : un Avertissement ( De la science du plain-chant»), un solfège (« De la connoissance des notes ») qui prend le parti du système 
moderne contre celui de la solmisation (notation, portée, clefs, nom des notes, bémol et bécarre - dont le « za » pour le si bémol -), intervalles - de la seconde à l'octave -, gammes, « manière de joindre la Lestre aux tons des Notes » (place des mots sous la musique), tons d'église (jamais plus de 8, chacun portant la qualification expressive courante jusqu'au $\mathrm{XIX}^{\mathrm{e}}$ siècle) et leur structure (intonation, ou " modulation », dominante, médiante ou " méditation », finale ou «conclusion »). Suivent de nombreux exemples pour les 8 tons. (Exemple 5)

La Méthode nouvelle pour apprendre parfaitement les règles du plain-chant et de la psalmodie, de Mr de La Feillee, Poitiers, première édition de 1748 ( $\left.\mathrm{n}^{\circ} 33\right)$ se retrouve en plusieurs exemplaires en Nouvelle-France. Cette méthode est une des plus considérables et des plus populaires du XVIII' siècle, aux côtés de celles de Lebeuf et de Poisson ${ }^{18}$ (que nous n'avons curieusement pas, à ce jour, retrouvées en Nouvelle France). Le plan suivi rappelle celui des sources précédentes. En distinguant le « plain-chant ordinaire » du « plain-chant figuré ou musical », il propose un système de valeurs plus complexe propre au plain-chant musical ( "longues » avec ou sans point, « quarrées » et « demi-quarrées » avec ou sans queue, grandes et petites «brèves »- losangées de tailles différentes, toutes valeurs variant avec les signes de mesures binaires ou ternaires). Les remarques sur l'art du chant sont nombreuses : « il faut des voix douces, faciles, justes, souples et légères » (p. 112) propres au plain-chant musical très orné (« sons filés », «cadences », « demi-cadence », «port-de-voix », dont la « chûte» (p. 118126)). À cela, il faut joindre des remarques sur la voix, la respiration, de même que sur l'expression et sur le tempo en fonction de la solennité de la fête. Cette méthode est suivie de messes composées dans les 8 tons, dont certaines font alterner le «plain-chant uni » (le chœur) et le plain-chant musical (le soliste), de motets (pour les principales fêtes de l'année dont celle du Saint-Sacrement ou de la Vierge), de vêpres et de Ténèbres dans la tradition de Nivers, Couperin ou Charpentier.

Hormis certaines fêtes comme celle de la Sainte-Famille, on aura remarqué la diversité presque décourageante des sources : la pratique était-elle cohérente? Quelle version mélodique et rythmique pouvait-on adopter? Dans ce monde musical où régnait la diversité, les chantres des Ursulines ont dû prendre des décisions en fonction de leurs connaissances et de leurs possibilités.

\section{L'ART DU CHANT DANS LES SOURCES}

\section{Chanter les louanges}

Au milieu de cette apparente incohérence, il reste une piste sûre : la raison d'être 18 Voir Lebeuf 1741 et Poisson 1750. 
de ce chant, qui est la louange, donc l'expression la plus sincère et la plus juste possible de la Parole. En effet, le chapitre "de l'oraison mentale " des constitutions de 1647 enseigne que l'amour doit conduire à « plaire à Dieu et glorifier sa divine Majesté». Le chapitre suivant, «De l'oraison vocale », qui fait appel « à l'employ des facultez extérieures » est la conséquence de ce principe premier :

Et d'autant que le tout aboutit à la parole qui fait la louange et à l'action qui fait l'honeur, ce chapitre est destiné, sous le nom d'oraison vocale, à l'establissement de l'usage de la voix et de la parole pour prononcer ou chanter les louanges de Dieu, comme les suivans chapitres sont consacrez aux actions capables d'honorer cette adorable Majesté. ${ }^{19}$

L'» oraison vocale » repose donc sur un acte d'abord affectif (l' » oraison mentale »), puis sur les divers degrés de « l'usage de la voix », qui va de la récitation privée à voix basse jusqu'au chant raffiné des motets. ${ }^{20}$

\section{2. « dire » ou « chanter »?}

Dans ce sens, les constitutions insisteront d'abord sur la juste prononciation des textes sacrés. Ainsi le Coutumier de Tours (1635) précise que la « mère du chant » fera « prononcer distinctement et clairement les mots latins, en chantant et disant l'Office [...] d'une voix plaine, plaintive et devote ». Il s'agit ici d'une prononciation des voyelles à la française, avec respect des accents toniques et peut-être de la quantité de prosodie latine. Cependant, les rédacteurs utilisent sans grande précision les expressions " dire ", " réciter 》 et " chanter ", auxquelles il faut joindre la fameuse « voix droite ». Je suggère ici une classification de ces divers sens :

- Premier sens général : « dire » = « officier » (« dire la messe »)

- Deuxième sens : « dire » = tout acte de la parole :

« Le Credo lorsqu'il doit être dit [aux périodes prévues par le calendrier liturgique] se chantera »

(Constitutions de 1647).

- Troisième sens : « dire » = « réciter » pour soi ou en chœur :

Quant aux litanies et au chapelet, il n'y a aucune obligation de s'assembler pour les dire $[\ldots]$ une chacune en fera en son particulier selon sa dévotion ${ }^{21}$

19 Constitutions, 1647, p. 52.

${ }^{20}$ On aura reconnu ici la loi de la convenance, principe premier de la rhétorique classique et de l'esthétique baroque.

${ }^{21}$ Constitutions, 1647, p. 149. 
[...]. Aux messes des morts solennelles si on peut chanter le chant convenable de l'Eglise on le fera, sinon on dira à voix droite ce qu'il faudrait chanter. $^{22}$

Le plus souvent, les constitutions marquent nettement la différence entre « dire » et « chanter ». Ainsi pour le Saint-Sacrement :

...elles chanteront tous les jours ouvriers, à l'élévation de la sainte hostie quelque motet en son honneur... ${ }^{23}$

La chaîne comprend donc tous les maillons possibles : simple récitation à voix basse, prédication du prêtre (sans doute modulée à la manière des orateurs de la chaire, rhétorique prévue par la Ratio Studiorum des Jésuites), récitatifs simples (litanies, psalmodie), chants de la messe plus ou moins complexe. C'est sur cet axe que, au gré des évêques, s'est déplacée, tantôt vers le « dire ", tantôt vers le " chanter », la pratique musicale des Ursulines aux XVII et XVIII ${ }^{e}$ siècles.

\section{La psalmodie}

La psalmodie la plus souvent décrite est celle que Mgr de Laval chercha à imposer, «psalmodie directe » ou « continue "24 réservée principalement aux Petites Heures :

Elles diront Prime, Tierce, Sexte et None du petit Office de Notre-Dame, tout de suite, le récitant alternativement en deux chœurs sans inflexion de voix, et y observant les médiantes, inclinations, et cérémonies ${ }^{25}$.

Cependant, cette psalmodie respecte la règle de la solennité de la fête. Comme les Statuts de Tours, 1661, les constitutions de Paris (1673), pourtant plus sévères, distinguent entre la «psalmodie solennelle » dite "d'un ton haut » (messe et Vêpres des dimanches et fêtes de première classe), du « ton moins haut » ou « plus bas » des fêtes de deuxième classe ou ordinaires.

\section{Le plain-chant proprement dit}

Il faut souligner que ce plain-chant ne concerne que certaines parties des fêtes relevées plus haut. Les constitutions de 1647 précisent que :

la messe se chantera en sorte que le prêtre dise de la messe haute tout ce qui a coutume d'être chanté par les prêtres à l'autel; mais pour elles [les

22 Ibid., p. 54.

${ }^{23}$ Ibid., p. 55.

24 "Lorsqu'on les [psaumes et cantiques] chante sans Antienne, c'est [...] à voix directe, de manière que cela ressemble plutôt à une simple récitation...", Jean Lebeuf (1741 : 179).

25 Constitutions de Bordeaux, 1635, p. 171. 
religieuses] au dedans elles ne chanteront que le Gloria in excelsis, et quand il ne doit point être dit, comme l'Avent ou le Carême, elles chanteront le Kyrie à sa place, le Credo lorsqu'il doit être dit se chantera; item le Sanctus devant [avant] l'élévation et le Benedictus après, le tout en chant d'Eglise, comme aussi ce que le chœur a coutume de répondre au prêtre; le reste de la messe se dira d'elles à voix droite.

C'est ce chant que Mgr de Laval ordonnera de supprimer et qui, on l'a vu, revint au XVIII' ${ }^{\mathrm{e}}$ siècle.

Par ailleurs, les sources posent avant tout le problème des valeurs, en rapport avec la quantité de la prosodie latine. Tant que la lumière n'aura pas été faite sur cette question de manière générale, il faudra s'en tenir à des hypothèses. Les pièces manuscrites en notation blanche ou les méthodes peuvent fournir certains éclaircissements. Ainsi, les Epitome de La Feillée, 1781 (graduel) et 1782 (antiphonaire) ( ${ }^{\text {os }} 24$ et 29) peuvent être mis en relation avec la méthode du même auteur (1748) $\left(\mathrm{n}^{\circ} 33\right)$. Par contre, les Cantus diversi de $1643\left(\mathrm{n}^{\circ} 23\right)$ font allusion à Nivers et Dumont, mais sont suivies de méthodes qui n'expliquent pas les valeurs...

\section{Les pièces en « simili plain-chant »}

Certains manuscrits, comme ceux consacrés aux vêtures et professions, proposent des pièces de type cantiques sur 4 ou 5 lignes. Parfois, la notation en blanches et noires modernes permet de reconstituer le rythme, qui respecte les accents toniques.

\section{Le plain-chant musical : la Messe Bordeloise et la Messe Royale : l'art du chant français}

La pratique d'un chant baroque quasi profane est attestée par les méthodes et les manuscrits qui mêlent plain-chant proprement dit (parfois en notation blanche), plain-chant musical, motets en notation blanche avec une ornementation à la francaise. Certaines sources contiennent des messes en plain-chant musical très orné, comme la Messe Bordeloise et la Messe Royale de Dumont, qui fournissent l'ordinaire de messes comme celle de la Sainte-Famille ou du Sacré-Cæur, auxquelles elles sont souvent jointes. Les manuscrits 8 et 9 sont des exemples remarquables de ce chant savant. (Exemple 6)

Valeurs : une comparaison entre les différentes versions de la Messe Bordeloise met en évidence la pratique non homogène du XVIII ${ }^{e}$ siècle. Ainsi, les manuscrits 6 et 8 mêlent sans grande précision longues, carrées et losangées, ces dernières 
étant visiblement considérées comme des " traits ». Par contre, l'Epitome gradualis romani de La Feillée $\left(\mathrm{n}^{0} 24\right)$ propose une version de la Messe Bordeloise où ces mêmes traits sont notés, dans la partie du « solus », en carrées et losangées alternées, ce qui implique un rythme en notes inégales évident, formant un contraste plein d'effet avec les carrées réservées au chœur.

Agréments : dans le manuscrit 8, le chœur (« chorus », marqué " gravement ») chante les Kyrie I et III et le Christe II en plain-chant « uni », avec quelques ornements (trilles marqués par une croix). Le « solus » (" gayement ») chante le Kyrie II et les Christe I et III avec de nombreux ornements et des liaisons soulignant les " passages ». Le Gloria repose sur la même alternance, avec l'introduction, au Solus, de ports-de-voix (appoggiatures) et de chutes et chutes de tierces notées par de petites croches liées.

Expression et tempo : les indications sont nombreuses et précises dans les manuscrits 6 et 8 . Les traditionnelles notes longues de " Jesu Christe " sont soulignées par l'expression « pesant doucement sur les notes ». Dans le Credo, la première entrée du chœur, après l'intonation du « célébrant » (« Patrem omnipotentem ») est marquée par « il faut bien battre les notes ». Le solus se réserve quelques traits quasi virtuoses sur « et resurrexit ».

Rhétorique musicale : les figures classiques ne sont pas rares, en particulier dans le Credo de la Messe Bordeloise ( $\mathrm{n}^{\circ}$ 9) : figure descendante sur «Descendit » (catabase) et figure montante sur «Et ascendit in caelum » (anabase), vocalise sur « Gloria », notes longues répétées sur « Mortuorum », etc. Cette virtuosité parcourt encore le Sanctus (vocalises du Gloria), alors que l'Angus Dei, rempli d'agréments, fait songer à la musique tendre des airs de cour et des cantates bucoliques françaises.

\section{Conclusion}

De nombreuses questions demeurent en suspens : versions mélodiques des pièces (dont les hymnes), modèles psalmodiques, rituels et relations avec les bréviares (nous n'avons pas retrouvé de cérémonial de chœur auquel les constitutions font allusion), alternance (chantres, chœur double, orgue, etc.), valeurs, usage réel des ornements, formation des voix, etc. Une recherche d'envergure sur le plain-chant gallican n'existe pas, qui permettrait de mieux poser les jalons des recherches locales, une des questions les plus épineuses étant celle de la classification et de la filiation des sources ${ }^{26}$.

${ }^{26}$ Le classique Graduel et Antiphonaire romain (1913) de Gastoué n'est plus qu'une bonne introduction à cette question. 
Il reste cependant que l'exemple des Ursulines permet d'éclairer quelque peu la pratique du plain-chant en Nouvelle-France (et sans doute en France elle-même) durant les $\mathrm{XVII}^{\mathrm{e}}$ et $\mathrm{XVIII}{ }^{\mathrm{e}}$ siècles. Les péripéties musicales des Ursulines démontrent en effet combien la tradition du chant va chercher à se maintenir : ne retrouverons-pas des noms de sœurs entrées au couvent de Québec vers 1920 dans des livres de chant arrivés deux siècles plus tôt ? Leurs aînées ont, pour certaines en tout cas, fait preuve d'une bonne compétence : les pièces en plainchant musical sont là pour le prouver. Dans l'ensemble de notre recherche sur le territoire de la Nouvelle-France, de Rimouski à Montréal, les sources conservées chez les Ursulines, par leur diversité et leur richesse, les placent parmi le «peloton de tête ».

Toutefois, le problème de la cohérence entre les sources met en évidence le fossé qui séparait, dans le monde baroque et gallican, la théorie de la pratique. Nous avons souligné combien les constitutions, toutes tendances confondues, parlent d'abord du dire et ensuite (peut-être) du chanter.

Il semble donc que cette " compétence » soit aussi d'un autre ordre : la connaissance des textes sacrés et le désir de plaire à Dieu. À l'image de la France gallicane, et au grand désespoir des théoriciens du temps, la France (donc la Nouvelle-France) ne semblait pas appliquer à la lettre des principes cohérents et communs. Cette unification et de la liturgie et de la pratique musicale ne viendra qu'avec la fin du XIX ${ }^{\mathrm{e}}$ siècle, soit avec la naissance de la musicologie. Étudier le plain-chant baroque nous ouvre ainsi certaines perspectives sur le «Performing practice » lui-même. Les divers champs d'étude dont le New Grove (« Performing practice ») dresse la liste (sources, notation, ornements, voix, etc.) réservent en fait une place bien mince à la raison même de la pratique musicale, qui est justement de dire, d'exprimer quelque chose (mais est-ce de la musicologie "sérieuse » ?). Pourtant, jamais la seule étude des moyens ne suffira à donner un sens à l'activité musicale. Par contre, comprendre cette expression, c'est retrouver le sens. Dans une lointaine et difficile Nouvelle-France, les Ursulines « ne firent pas de la musique » : discrètement, mais avec ténacité, elles s'efforcèrent de choisir, de conserver et d'entretenir ce qui leur semblait être les meilleurs moyens alors possibles (et permis) de chanter leur passion.

\section{BIBLIOGRAPHIE}

\section{A. Sources musicales}

Les titres des manuscrits proviennent le plus souvent de la première, ceux des imprimés ont été abrégés. La numérotation ne vaut que pour le présent article. Dans la mesure du possible, les dimensions et nombre de pages ont été précisés pour les manuscrits. Les 
sources sont localisées aux Archives des Ursulines de Québec. Le sigle AUTR indique une localisation aux Archives des Ursulines de Trois-Rivières.

\section{MANUSCRITS}

Deux manuscrits comparables, que la fête de sainte Angèle (béatifiée en 1769) permet de dater de la fin du XVIII ${ }^{e}$ siècle :

(1) Vêpres de Lavent [Avent], $13 \times 17,5 \mathrm{~cm}, 276 \mathrm{p}$. (Rois, Circoncision, dimanche de la Passion, Rameaux, Triduum pascal, dimanche de Pâques, Vêpres de la SainteFamille, commun des saints). À rapprocher des manuscrits Sainte-Famille type I.

(2) Pour la fête de Saint Joseph antiennes, $16 \times 20,5 \mathrm{~cm}, 30 \mathrm{p}$. (Ténèbres, sainte Angèle, Assomption, saint Hyacinthe, saint Jean, saints Martyres, saint Augustin).

Deux manuscrits cherchant à imiter les caractères imprimés, et d'aspect semblable:

(3) Lectiones Tridui Ante Pascha (Lamentations de Jérémie). Oblong, $20 \times 28 \mathrm{~cm}, 20$ p. Page de garde : « Noviciat », Ornements et liaisons rajoutés.

(4) In Festo Sanctissimae Familiae Jesu Mariae Joseph (en réalité : fête de saint Joseph). $19 \times 24 \mathrm{~cm},[20$ p.].

Une copie d'un type de recueil imprimé, longtemps populaire :

(5) Cantus diversi prodominicis et festisper annum, copie manuscrite par Jean François Wade, 1749, ayant appartenu à Roland Braux (1749). 10,5×15,5cm, 119 p. (AUTR).

Manuscrits remarquables pour leur plain-chant musical très orné :

(6) Livre cantoral de plain-chant pour les Rses Ursulines de Québec Nouvelle France qui sont très humblement Suppliées de prier Dieu pour celle qui leur présente ce livre Pour leur usage. $15,5 \times 20,5 \mathrm{~cm}$ [154 p.] (« À l'usage de Ste Augustine de la Conception »-Ursuline de Paris). Office de Noël, Cendres, Rameaux, Semaine sainte dont Ténèbres, Prose Veni Sancte Spiritus, antiennes et hymnes pour les fêtes des Ursulines, dont sainte Ursule et saint Augustin, Te deum. L'office de la Sainte-Famille a été rajouté par une autre main. Nombreuses indications sur « ce qui se chante » ou « se chante encore ». Notation carrée et notation blanche.

(7) Missa Vulgo Bordeloise (et messes Sainte-Famille, Saint Nom de Jésus, SacréCœur, Messe Royale de Dumont, Credo Pujol, motets à 2 voix en notation moderne, Martyrologe pour Noël). 1785 sur la page de garde. $23 \times 21 \mathrm{~cm}, 77 \mathrm{p}$. (Les messes sont très semblables au manuscrit précédent : même main, même notation).

(8) Messe du Sacré Cour de Jésus (Messe Bordeloise, hymne à sainte Ursule, messe de la Sainte-Famille, de l'Esprit saint, du 6e ton [H. Dumont], motets à la Vierge en notation blanche avec ornements, cantiques en français, dont sur un sur une musette de Rameau (Pièces de clavecin, 1724). 16,5 × 19,5cm. «Maire [mère] D... rel[igieuse] ». En fait, un ensemble de manuscrits de mains et de notations diverses, avec nombreux ornements, liaisons et indications d'alternance. Date possible : 1785.

(9) Cayer des Antiennes, Messes du Sacré-Cœur et Bordeloise, Antiennes pour le 
Tems de Lavent et du Carême 1793 avec antiennes du Sacré-Cæur, de saint Augustin, de sainte Ursule (Vêpres), hymne (2 voix), motet et répons au SaintSacrement, motet à la Vierge, Vêpres de la Sainte-Famille (sans musique), Motets du Saint-Sacrement). Première page : «Sr Saint Thomas avec permission de la sainte obéissance (...) 1793 » (permission de la mère supérieure). Sœur Saint Thomas est entrée aux Ursulines de Québec en 1783 et mourut en 1804).

\section{Office de la Sainte-Famille}

Cet office se retrouve dans certains manuscrits ou imprimés et dans les manuscrits suivants, consacrés exclusivement à ladite fête (manuscrits très voisins) :

(10) Messes et Vêpres pour le Saint Nom de Jésus - sans musique (Avec Messe et Vêpres pour la Sainte-Famille). $20 \times 26 \mathrm{~cm}$, [ $30 \mathrm{p}$.]. Seule la séquence de la SainteFamille est notée. Est-ce le recueil que Glandelet a « composé » pourl'enseignement des Ursulines (cf. Annales, 1700)?

(11) Messe de la Sainte-Famille, $18 \times 23 \mathrm{~cm},[4 \mathrm{p}$.], notation blanche et noire moderne sur 4 lignes. Seule la séquence est notée.

(12) Séquence de la Sainte-Famille, $18 \times 23,5 \mathrm{~cm}$, [4 p.], très semblable à :

(13) Séquence de la Sainte-Famille, signée "Sœur Saint Joseph R.L. qu[ébec?]», oblong 18,5 $\times 19,5 \mathrm{~cm},[16 \mathrm{p}$.]. portée rouge. Six sœurs Saint Joseph se sont succédées. La première, arrivée de Tours en 1639, morte en 1652, est improbable. Les autres vont de 1671 à 1842 .

3. Manuscrits de motets et cantiques (notation moderne) contenant du plain-chant (notation carrée)

(14) Motet au Saint-Sacrement... (dont messe du 6e ton), 15,5 × 20,5 cm, [225 p.].

(15) Cantiques en deux parties pour Noël (avec une hymne au Saint-Sacrement), $10 \times$ $16 \mathrm{~cm},[271 \mathrm{p}$.$] .$

\section{Vêtures et professions (AUTR seulement)}

(16) Cérémonial des vêtures et professions Pour les Religieuses de Sainte Ursule de la Congrégation de Paris. Paris : Louis Josse, 1716 (approbation 1625). La musique (plus tardive) se trouve dans deux manuscrits de mains différentes:

(17) Cérémonial des vêtures et professions Pour les Religieuses de Sainte Ursule de la Congrégation de Paris, deux manuscrits, $15 \times 20 \mathrm{~cm}$ et $16.5 \times 20,5 \mathrm{~cm}$.

(18) Cérémonial des Vêtures et Professions pour les religieuses de Sainte-Ursule de la Congrégation de Paris, nouvelle édition de 1860 (Clermont-Ferrand), et une autre de 1893 , textes qui reprennent en fait l'édition de 1716 , mais sans la musique. Une troisième édition (Ursulines de Québec), due à l'Abbaye de Solesmes (1897), propose une nouvelle version musicale.

\section{Sépultures}

(19) Cérémonial des sacrements... Ordre de la sépulture, Paris : Eloy Elie, 1681 (approbation 1656). La musique se trouve dans les Cantus diversi de 1643 (AUTR) (no 23). 
(20) Cérémonial des sacrements... Ordre de la sépulture (AUTR), $16 \times 20 \mathrm{~cm}$. Copie manuscrite (début $\mathrm{XIX}^{\mathrm{e}} \mathrm{s}$. ?) du précédent.

\section{IMPRIMÉS}

\section{Graduels}

(21) Graduale romanum.Paris: Christophe Ballard, 1697 (approbation Dumont, Robert, Nivers, 1682). Signature de sœur Sainte Augustine, 1750 (entrée 1737, morte 1790). La fête du Sacré-Cœur à été ajoutée à la main.

(22) Graduale romanum. Paris : Nicolas Pepie, 1706 (approbation de Dumont et Robert 1682, censure de Nivers 1706). 20 pages manuscrites ajoutées à la fin (Messes Royale, du 2e ton, du Sacré-Cœur, Bordeloise, du Saint-Sacrement et de la Vierge).

(23) Cantus diversi, Lyon : B. Martin. 1643 (relié avec Trois mêthodesfaciles..., 1713) (approbation Dumont et Nivers) (AUTR).

(24) La Feillée, Epitome gradualis romani... A Dom. De la Feilée revisum, auctum et emendum, Poitiers : Jean-Félix Faulcon, 1781 (privilège 1780). D'après le Graduel etl'Antiphonaire de Nivers, 1697. (Messes Royale, Impériale, Thérique, Bordeloise, etc.).

\section{Antiphonaires}

(25) Antiphonarium romanum. Paris : Jean de La Caille, 1666 (privilège : 1663).

(26) G.G. Nivers, Antiphonarium romanum... in usum et gratiam Monialium Ordinis S.P. Augustini... Opera et Studio Guilielmi Grabrielis Nivers... Paris : Robert Ballard, 1671 (avec rajouts manuscrits, en particulier, de la séquence de la SainteFamille et de l'introït Gaudeamus utilisé pour la même messe).

(27) Antiphonarium juxta breviarium. Lyon : B. Martin, 1724 (privilège 1722).

(28) Epitome Antiphonarii romani seu Vesperale. Vannes : Jean Nicolas Galles, 1773 (privilège 1770) (Avertissement : "Conforme aux dernières éditions des grands livres de chœur imprimés à Lyon »). Cet exemplaire comprend, dans les lectures du Jeudi saint, les noms de sœurs de la deuxième moitié du XVIII ${ }^{\mathrm{e}}$ siècle et du $\mathrm{XX}^{\mathrm{e}}$ siècle.

(29) La Feillée, Epitome Antiphonarii seu Vesperale. Poitiers : Jean-Félix Faulcon, 1782 (approbation 1772, privilège 1780). Fait référence à Nivers.

\section{Méthodes}

(30) Nouvelle méthode pour apprendre le plain-chant. Paris : G. Desprez, 1683 (imprimerie Ballard). La page-titre manque. Original : Paris, Bibliothèque nationale, Rés. 2237). Est-ce la méthode de « Mr Drouault » dont parle La Feillée dans sa Méthode nouvelle, édition de 1754. p. 15 ?

(31) „Observationes » du Graduale romanum. Paris : Christophe Ballard, 1697 (voir $\left.\mathrm{n}^{\circ} 21\right)$.

(32) Trois méthodes de plain-chant. Lyon: B. Martin, 1713, relié avec les Cantu diversi, 1643 (AUTR), voir $n^{\circ} 23$.

(33) La Feillée. Méthode nouvelle pour apprendre... le plain-chant. Poitiers : Faulcon, 1748 (première édition). Deux exemplaires. 
(34) A Essay or Instructions for learning the Church Plain-chant. Londres : J.P. Coghlan, 1799 (« Thomas Maguire 1803 » [chapelain des Ursulines de TroisRivières vers 1835]).

\section{Divers}

(35) Passiones quatuor, Paris, 1691 (venu des Ursulines de Paris en 1835).

(36) Psautier : Bréviaire, partie d' hiver. Paris, 1645.

(37) Offices de la Semaine sainte (peu de musique), Paris, 1651, 1662, 1680, 1688, $1699,1700,1717,1728$, etc.

(38) Bréviaires : Bréviaire romain, Paris, 1665, Diurnal du Bréviare romain pour les Ursulines, Paris, 1714 et 1729.

\section{Après 1800}

Dès le début du $\mathrm{X} 1 \mathrm{X}^{\mathrm{e}}$ siècle, les Ursulines possèdent les recueils imprimés en NouvelleFrance, démontrant ainsi la continuité musicale de leur pratique. On relève ainsi des ouvrages (et leurs éditions successives) comme le Graduel romain de 1800, ou le Processionnal romain, 1801, imprimés chez Neilson à Québec.

\section{B. RÉFÉRENCES}

\section{ANNALES ET RÈGLEMENTS}

\section{[ANONYME]}

1639-1822: Annales des Ursulines de Québec, tome I, manuscrit, Archives des Ursulines de Québec.

\section{[ANONYME]}

1635 : Les Constitutions de l'Institut et compagnie des religieuses de SainteUrsule, qui doivent être observées de toutes celles qui y sont et y entreront, avec : Coutumier des Religieuses Ursulines, dans lequel sont contenues, et amplement déclarées les Constitutions ci-dessus approuvées: Et ordonnées par Monsieur l' Archevêque de Tours. Paris : Georges Josse, 1635. (Constitutions dites de Bordeaux-Tours).

\section{LALEMANT P.J.}

1647 : Constitutions et règlements des premières ursulines de Québec. Québec : manuscrit. Édition moderne : Sr. Gabrielle Lapointe, Québec : Monastère des Ursulines, 1974.

\section{[ANONYME]}

1661 : Statuts des religieuses Ursulines de la Ville et du Diocèse de Tours. Paris : [?], 1661.

\section{[ANONYME]}

1876(1673): Règle de Nostre Père S. Augustin, suivi de : Les Constitutions du monastère de Saincte Ursule de Paris : François Jacquin (approbation : 1640). 
L'exemplaire du monastère de Québec daté de 1876 est une réédition de la publication de 1673 .

\section{DIVERS}

\section{LEBEUF, Jean}

1741 : Traité historique et pratique sur le chant ecclésiastique.Paris :J.B. Hérissant.

\section{OURY, Dom Guy}

1971 : Marie de l'Incarnation (1599-1672). Correspondance. Solesmes : Abbaye Saint-Pierre.

POISSON, Léonard

1750 : Traité historique et pratique du plain-chant. Paris : Ph.N. Lottin et J.H. Butard.

POULIOT, Adrien

1980: «Une primeur québécoise : la fête et l'office de la Sainte-Famille ", Symposium de Joséphologie, Montréal (tiré à part).

PRUITT, William

1973 : « Bibliographie des œuvres de Guillaume Gabriel Nivers ». Recherches sur la musique francaise, XIII, 133-156.

\section{SAINTE THÉRÈSE DE LISIEUX, mère}

1961 : Petite histoire de la liturgie et du chant grégorien au monastère des Ursulines de Trois-Riviéres, document dactylographié. Trois-Rivières: Monastère des Ursulines.

\section{SCHWANDT, Erich}

1981 : "The Motet in New France : some $17^{\text {th }}$ and $18^{\text {th }}$ Century Manuscripts in Quebec ». Fontes Artis Musicae, 28.

1984/1 : «Le motet classique français en Nouvelle-France : cent années d'adaptation (1652-1755) ». Actes du colloque international de musicologie sur le grand motet francais (1663-1792). Paris : Centre d'études de la musique française aux XVIII ${ }^{e}$ et XIX ${ }^{e}$ siècles.

1984/2 : "Les vêpres en Nouvelle-France : musique et liturgie ", Les Cahiers de l'ARMuQ, Montréal/Québec. 
4ै 4 w uxcos

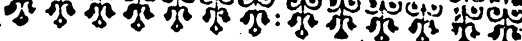

\section{LAMENTATIONES} JEREMIE PROPHEFE FERIA V. IN COENA DOMINI
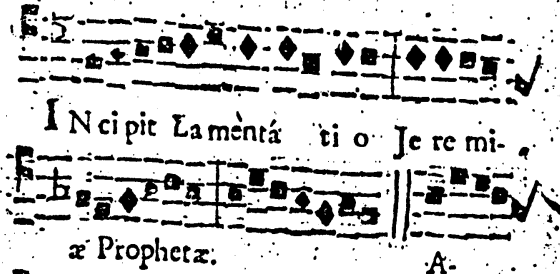

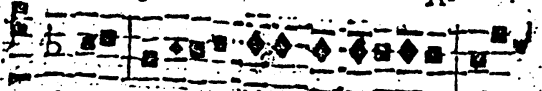

lepho Qiromodo fedet fo las civitas ple:

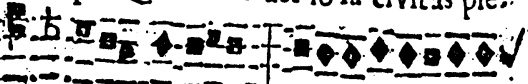

34 ! na populo; facta eft quafi vidua

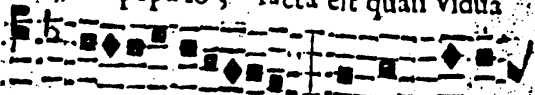
If domina Gen tium; Princeps provin
'Firid quinta in Cana Domitri ${ }^{2} g$

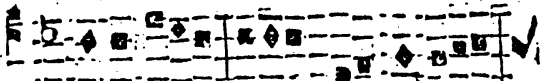

cis. rum factient lub tri bu to.

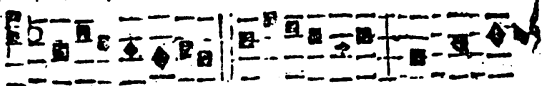

Beth. : Plorans plo:

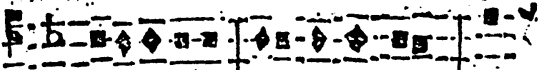

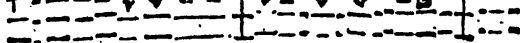

ravit in node, \&lachrymx ejus in

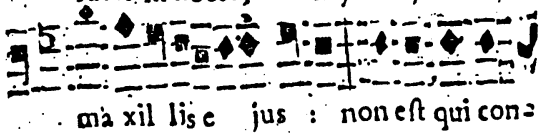

F:

fole tur e ana ex om ni buscharis

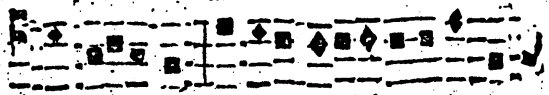
e- jus, Ornines a mici ejus lperve.

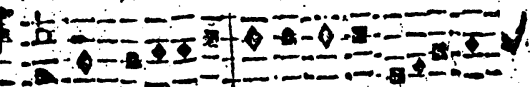
runt co.am, \& faqtifunt e $\mathrm{i}$ i in.

Hen mis cir Gimeli Migray siii

1. Lamentations de Jérémie, par G.G. Nivers, Cantus diversi, Lyon, 1643. 


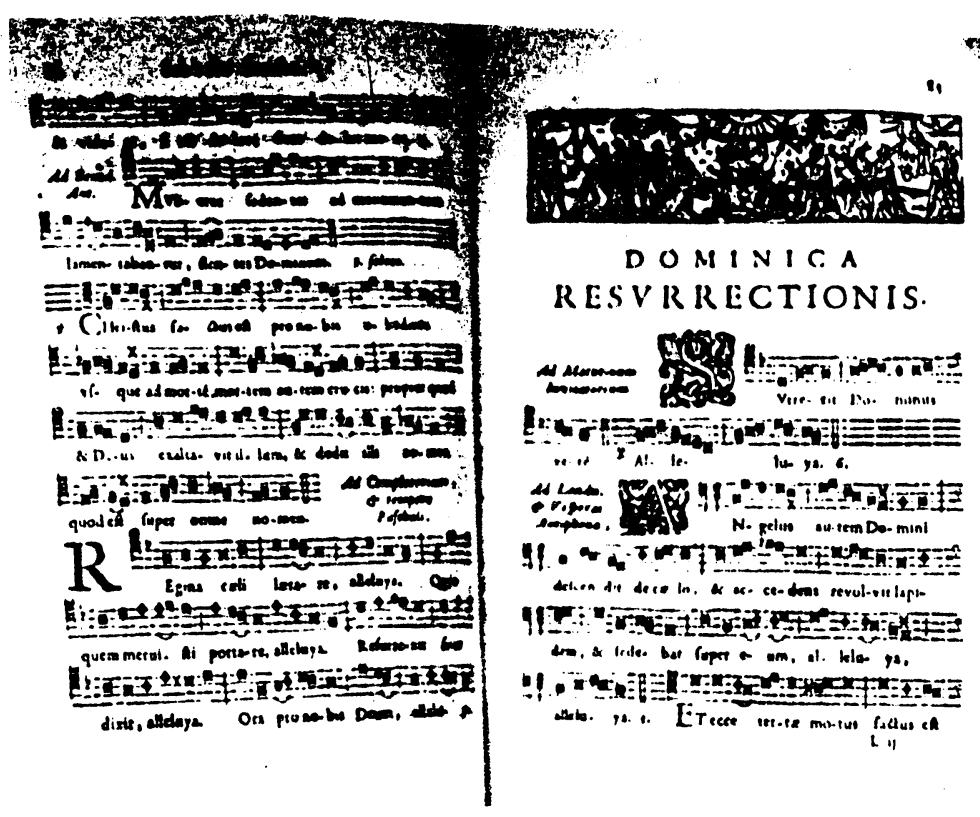

2. Guillaume Gabriel Nivers, Antiphonarium romanum, Paris, 1671. 
.... MISSA

Familia

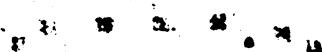

INTROITUS.

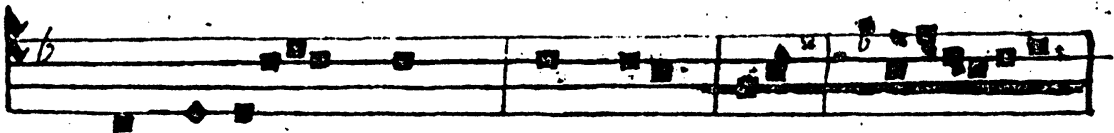

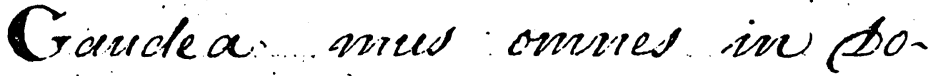

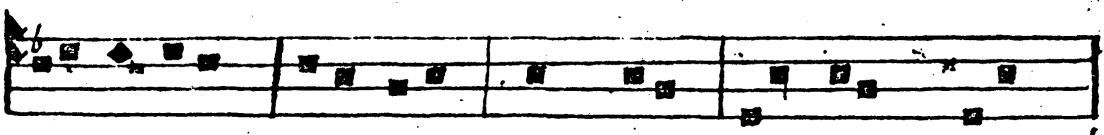

mino di eno fesilume fe-fe-bracin

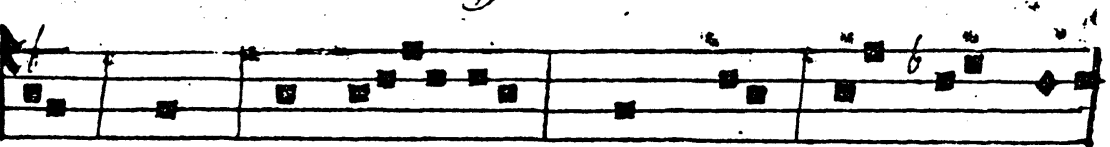

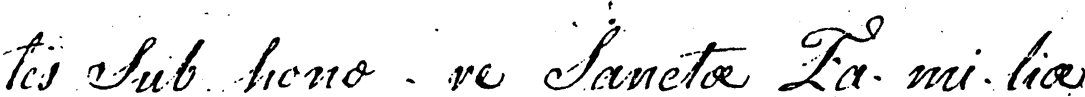

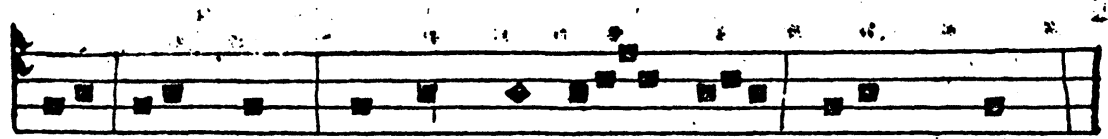
de enjus folenvinita te qau dent

3. Messe de la Sainte-Famille. Mansuscrit Messe du Sacré-Coeur de Jésus (XVIII siècle, annexe $n^{\circ} 8$ ). 


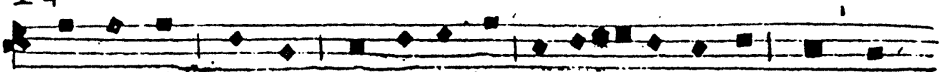

Glo-ri-a Pa.tri et Fi.li-o et Spi-ri-tu-i Sancato... Le chiceur reprend Quem vidi quem amavi.\&c. Le repons etant dit. onlus otera son cierge qui ne lui sera rendu, qui apres avoir salue los Religriau. ses et le celebrant se meitant a genoux com mence I hymne Veni creator pendant lequel la Novice sera prosmrnea les bras en croix corn mi: aursi durantlas souffrages et oraisonas. suivarite? Les choeurs le chanteront alternatu? rient. le cote drit: repondra: mentes tuorum \& on aura.soin de chanter cet Hynne ass zy : Veni cre.a-tor spi-ri-tu mentes tuorum visi ta im.
ple sü-perna gra-ti-a quetucre asti pectora.

\section{2}

Qui diceris Paraclitus, Altissimi donum dei, Fons vivus, ignis caritas, Et spiritabio urictio. Tu septiformis munere Digitus paternæe dexteræ si Tu rite promissum patris Sermone ditans guttura.

4. Cérémonial des vêtures et professions pour les Religieuses de Sainte Ursule de la Congrégation de Paris, copie manuscrite, début du $\mathrm{XIX}^{\mathrm{e}}$ siècle. 
196

Metbode du Ploin-Chanc.

Si l'on chante par binol, ilfaut prendeq fol, fur la ligne de la clef, parce qu'en $C$, fol, we, il y a fol, quil fe chante par binol, fur l'efpace au dellous il fsut piendre $f a, p s$ s. $^{2}$ ce quien $B, f a, f$, il $y$ a fis quii le clasire par bmol, \&c.

C'eft exemple fuffit pour faire connoitre les notes par le moyen de la Game : car auf: firtôt que l'on connoit les clefs, ou connoif en même-tems les notes en difaut la Game en montant ou en defcendsant, felon quẹ Les notes montelif ou delcendent.

- Lentonnement des notes eft le méms que cidevant, aumi bien que la manice dy joindre la leftefo à leurs Tons, $\because .$.

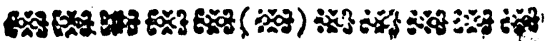

LES DIVERS T ONS $\therefore$ ou Intonnaciond des c bants comp

- muas de l'Egife.

T E mor'de Tonns fe prend pos ici pout Li li diftance qui fe tinưve enere les no: tes 2 corame on l'a pris cy devaht : mais. on entend certairres efpeces de Cliants, auf. quell; se peuvent rapporter tout ce qui la charse dansl'Eglife, Coir p foawmes Ansist
Divers Tons des Cbants comm. dol Eg.i2 mes Introirs, \& c. Os une regle qui faie connoitse rouse forte de Chants Ecclefiantis ques.

Il y en a huite, \& rien ne fe chante gui dile Coir'de quelqui in de ces huit Tons.

Qn les diftingue par leur fon ou harj monis qui en differente dans chacun, ainfi qu'ou le voir par ces Epircheres qu'on leur a: donicicos.

$$
\begin{aligned}
& \text { Primus - Gravis. } \\
& \text { Secundus - Triffie. } \\
& \text { Terrius - M. Ricus. } \\
& \text { Quariws - Horingrionfi } \\
& \text { Diminsus - Lasus. } \\
& \text { Stxsius - Divorus. } \\
& \text { 2. Seprinas - Angelicus. } \\
& \text { Ogtavms merfeltus. }
\end{aligned}
$$

On les corinnit par le moyen des Finales \& des Domina rices.

On appelle finale la note par laquelle. une Ansienne, Ripous ; \&c. finit.

La Doshinante eft celle qui fe nomime le. plus fouvańt dans un Répons, Introit Gras. duel, \&ec.

On voic darts la Table fuivante les Finaf les \& les Dominautes de chaque. Ton.

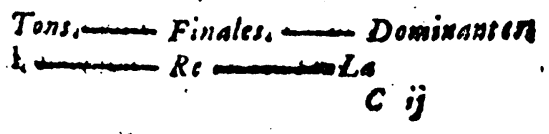

5. Méthode de plain-chant, Lyon, 1713 (relié avec les Cantus diversi, Lyon, 1713). 

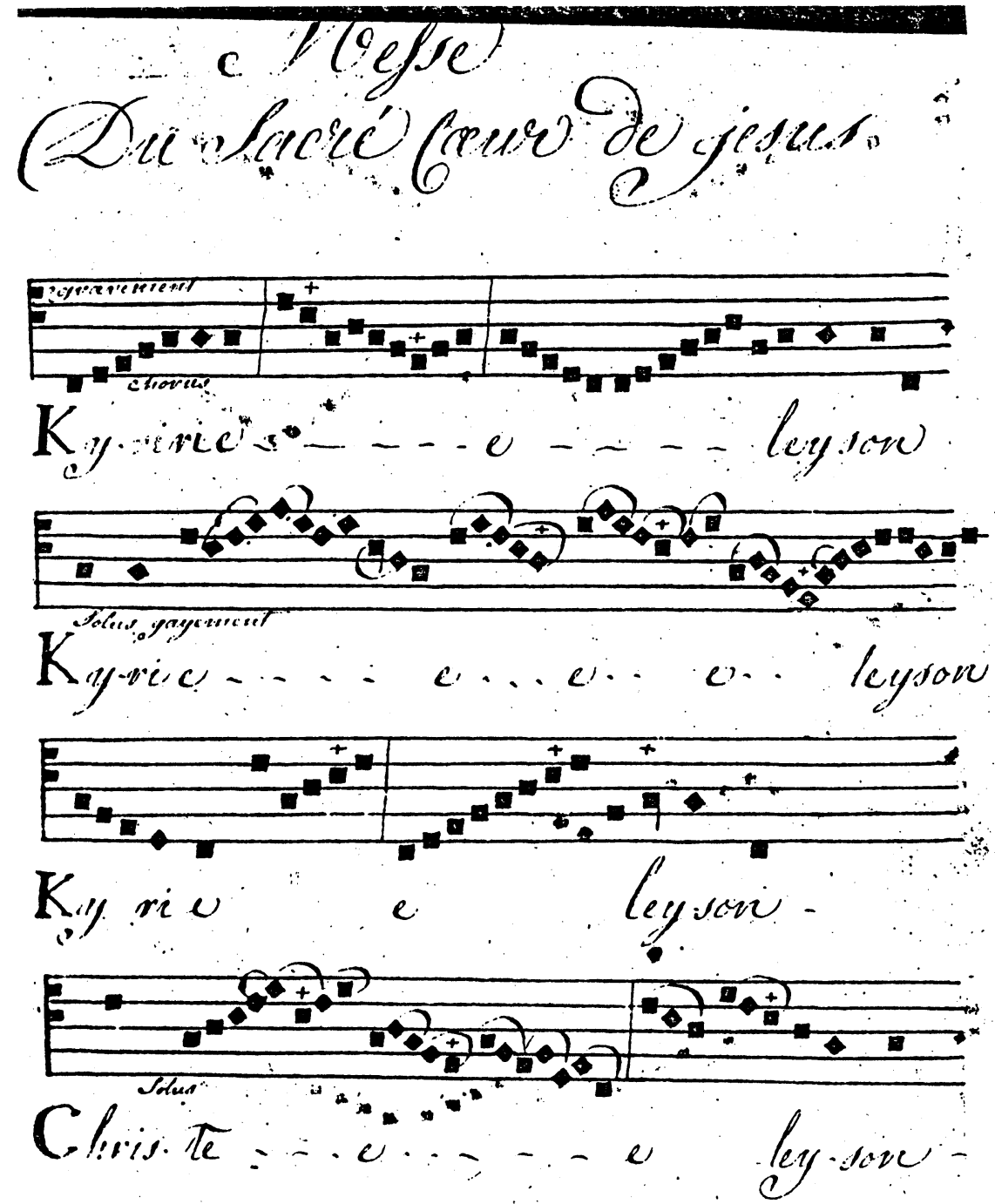

6. Messe Bordeloise. Mansuscrit Messe du Sacré-Couer de Jésus (XVIII siècle, annexe $\left.\mathrm{n}^{\circ} 8\right)$. 